\title{
Progress in Clinical Neurosciences: A Forum on the Early Management of Parkinson's Disease
}

\author{
Anthony E. Lang, Janis Miyasaki, C. Warren Olanow, A. Jon Stoessl, \\ Oksana Suchowersky
}

\begin{abstract}
There are numerous concerns related to treatment choices involving early dopaminergic therapy in Parkinson's disease. These include the effect on the underlying progression of the neurodegenerative process as well as the development of motor complications such as fluctuations and dyskinesias. A number of recent basic and clinical studies have provided new insights but have also added confusion and controversy. This report summarizes presentations and discussion dealing with these issues from a one-day symposium involving Canadian Movement Disorders neurologists.
\end{abstract}

RÉSUMÉ: Forum sur la prise en charge précoce de la maladie de Parkinson. Le traitement précoce de la maladie de Parkinson au moyen d'agents dopaminergiques soulève plusieurs questions, entre autres son effet sur la progression du processus neurodégénératif et sur le développement de complications motrices telles les fluctuations et les dyskinésies. Des études fondamentales et cliniques récentes ont fourni de nouvelles avenues de réflexion, mais elles ont également suscité la confusion et la controverse. Cet article résume les présentations et les discussions sur le sujet lors d'un symposium d'une journée tenue par des neurologues intéressés par les troubles du mouvement (Canadian Movement Disorders Group).

Can. J. Neurol. Sci. 2005; 32: 277-286

Over the past five to ten years there have been important developments impacting on the initiation of dopaminergic therapy in early Parkinson's disease. Critical concerns relate to the potential neurotoxic effects of levodopa as well as the all too common development of motor complications. There have been significant advances in our understanding of the pathogenesis of motor complications, particularly levodopa-induced dyskinesias. A number of studies have evaluated clinical outcomes of initiating levodopa as well as comparative outcomes of early therapy with dopamine agonists versus levodopa. A variety of claims have been made with respect to the impact of these treatments on the underlying disease progression, particularly based on modern imaging techniques. There are a number of outstanding questions or sources of controversy in this literature. In order to review the "state-of-the-art" and address these critical issues a group of Canadian Movement Disorders experts met together for a one-day symposium in Toronto on May 8, 2004 funded by an unrestricted educational grant by Novartis, Canada. Four speakers were chosen to review the pertinent literature and to highlight and address the areas of uncertainty and sources of controversy. An open discussion with input from all participants followed each presentation and the discussion was recorded for subsequent review. The following reports summarize the presentations and discussion that took place at the meeting.

\section{Pathogenesis of Motor Complications in Parkinson's Disease: New Approaches}

CW Olanow

Levodopa therapy remains the "gold standard" for the symptomatic therapy of Parkinson's disease (PD). However, long-term treatment is associated with the development of potentially disabling motor complications ${ }^{1}$. This has limited the value of levodopa therapy and is largely responsible for the resurgence of surgical therapies in PD. Indeed, no medical or surgical treatment has been shown to provide superior efficacy to levodopa. There are non-dopaminergic features that limit the potential of levodopa therapy (e.g. dementia, autonomic disturbances, and postural instability). However, the ability to deliver levodopa so as to provide symptomatic benefits without

\footnotetext{
From the Division of Neurology, Department of Medicine (AEL, JM), University of Toronto, Toronto Western Hospital; Department of Neurology (CWO), Mount Sinai School of Medicine,Toronto, Ontario; Pacific Parkinson's Research Center (AJS), University of British Columbia, Vancouver, British Columbia; Department of Clinical Neurosciences (OS), University of Calgary, Calgary, Alberta, Canada Received JANUARY 7, 2005. ACCEPTED IN FINAL FORM MARCh 22, 2005. Reprint requests to: Anthony Lang, Director, Movement Disorders Center, Toronto Western Hospital, 399 Bathurst St.,7McL, Toronto, ON M5T 2S8 Canada
} 
complicating dyskinesia or motor fluctuations would represent a major advance in the treatment of PD.

\section{Motor Complications}

Increasing evidence indicates that levodopa-induced motor complications are related to abnormal pulsatile stimulation of striatal dopamine receptors. ${ }^{2}$ Normally, dopamine neurons in the substantia nigra pars compacta $(\mathrm{SNc})$ fire continuously, and the firing rate is not modified by the presence or absence of movement. ${ }^{3}$ Thus striatal dopamine levels are stable and dopamine receptors are exposed to relatively constant levels of dopamine. Substantia nigra pars compacta dopamine neurons do burst in response to reward or increases in glutamate activation, ${ }^{4}$ but terminal dopamine reuptake is sufficiently robust that synaptic dopamine levels remain constant.

In PD, because there is a loss of substantia nigra pars compacta dopamine neurons, striatal dopamine levels are dependent upon the peripheral availability of exogenously administered levodopa. There have been no studies that establish how levodopa should be administered in order to provide dopamine to the brain in a physiologic way. Indeed, the recently completed ELLDOPA study (as discussed by Dr. Suchowersky) is the first double blind, controlled dose ranging study of levodopa that has been performed to date..$^{5}$

There is now considerable evidence indicating that pulsatile stimulation of dopamine receptors leads to gene and protein changes in striatal neurons with alterations in neuronal firing patterns and the consequent development of motor complications. ${ }^{2,6}$ Two factors contribute to the likelihood that pulsatile stimulation will occur; disease severity and half life of the dopaminergic agent employed. With greater disease severity, there is greater degeneration of striatal dopamine terminals and a reduction in their capacity to buffer fluctuations in striatal dopamine levels. Indeed, levodopa-induced dyskinesia emerge within days in MPTP monkeys where there is a 95\% loss of nigral neurons, whereas they develop over months or years in PD patients where there is typically a 30-60\% loss of dopamine neurons at the time symptoms first appear. Studies in MPTPlesioned monkeys also illustrate the importance of plasma half life in the induction of dyskinesia. ${ }^{7,8}$ Short-acting dopaminergic agents such as levodopa provide benefit that is associated with dyskinesia. In contrast, long-acting dopamine agonists provide comparable benefit, but with a marked reduction in both the severity and frequency of dyskinesia. Indeed, dyskinesia are seen with pulsatile administration of a short-acting dopamine agonist, but not when the same agent is administered by continuous infusion. ${ }^{9}$

These studies suggest that therapies that provide more constant activation of dopamine receptors (Continuous Dopaminergic Stimulation) might be associated with reduced motor complications. Several prospective, randomized, doubleblind, controlled studies have been performed in early untreated PD patients, comparing initial therapy with a short-acting formulation of levodopa and a long-acting dopamine agonist. ${ }^{10,11}$ Each study showed reduced motor complications in patients started on therapy with the dopamine agonist, even when initial therapy was supplemented with open label levodopa (as discussed by Dr. Miyasaki).
These results support the early use of dopamine agonists in the treatment of early PD patients. However, patients randomized to levodopa in these studies have improved UPDRS motor scores compared to patients treated with dopamine agonists. Further, virtually all PD patients eventually require levodopa. The ropinirole study showed that patients receiving dopamine agonist monotherapy had hardly any motor complications, while supplemental levodopa increased the frequency of motor complications even in the presence of a dopamine agonist. ${ }^{10}$ Indeed, the risk of developing dyskinesia is the same regardless of whether levodopa is introduced as initial therapy or as a supplement to the dopamine agonist (Personal communication - O Rascol).

It is thus evident that PD patients require levodopa, and that levodopa is associated with an increased risk of motor complications regardless of whether or not the patient is also taking a dopamine agonist. A key question is whether or not the risk of developing motor complications with levodopa could be reduced if the drug was administered in a longer-acting formulation. Continuous infusion of levodopa is known to be associated with reduced off time and reduced dyskinesia in comparison to the standard oral formulations of the drug. ${ }^{12-19} \mathrm{We}$ recently confirmed this observation and performed pharmacokinetic $(\mathrm{PK})$ studies when the patients were receiving oral levodopa and experiencing severe motor complications and then when they were receiving levodopa by infusion with a marked reduction in motor complications. ${ }^{20}$ The major distinction in these two PK patterns was that oral levodopa was associated with very low trough levels, while continuous infusion largely eliminated the low trough levels seen with oral delivery. These low trough levels likely represent periods during which striatal dopamine levels are reduced and striatal dopamine receptors are not activated, and thus may represent the pharmacologic basis of pulsatile stimulation. This PK pattern can serve as a template for designing an oral treatment strategy that models infusion. If an oral treatment strategy can be developed that mirrors the PK pattern of a continuous levodopa infusion, it is reasonable to consider that comparable clinical benefits with reduced motor complications might be obtained.

Frequent doses of regular formulations of levodopa (up to hourly) and controlled release formulations of levodopa do not prevent the development of low plasma levodopa trough levels (Stocchi and Olanow, unpublished data). We have, however, been able to avoid low trough levels and simulate the PK pattern of levodopa infusion by administering levodopa in combination with a catechol-O-methyltransferase (COMT) inhibitor. ${ }^{21}$ Catechol-O-methyltransferase is the major metabolic pathway for levodopa in the presence of a decarboxylase inhibitor, converting approximately $90 \%$ of orally administered levodopa to the inactive product 3-O-methyldopa. ${ }^{22}$ The addition of a COMT inhibitor increases the elimination half-life of levodopa, and thus reduces its potential to induce pulsatility. In MPTPlesioned monkeys, administration of levodopa in combination with entacapone significantly reduces the risk of dyskinesia in comparison to when levodopa is administered alone..$^{23}$

These studies collectively suggest that, in PD patients, it may be preferable to initiate levodopa therapy (given with an aromatic acid decarboxylase inhibitor) in combination with a COMT inhibitor in order to obtain maximal efficacy coupled 
with reduced risk of motor complications. At this time there is no evidence from clinical trials that this combination will reduce the risk of motor complications. To test this hypothesis, we have organized a prospective, double blind, clinical trial (STRIDE$\mathrm{PD})$. In this study, PD patients who require levodopa/carbidopa will be randomized to receive the drug in combination with entacapone or placebo. Patients will be included who are, and who are not, on dopamine agonists. Positive results in this study will support the introduction of levodopa therapy in combination with a COMT inhibitor and will likely influence the way PD patients are managed in the future.

\section{Levodopa therapy in early PD}

\section{O Suchowersky}

Since its introduction in the 1960s, levodopa has been recognized to be the most effective treatment for Parkinson's disease. ${ }^{24,25}$ All major motor symptoms show significant improvement, as do activities of daily living and quality of life, and it remains the most effective treatment over the course of the disease. Additionally, use of levodopa has been shown to increase life expectancy. Untreated PD patients have a life expectancy of under 10 years; ${ }^{26}{ }^{27}$ the advent of levodopa has reduced this mortality by half ${ }^{28}$ and longevity was increased in those who began levodopa earlier, at a time of less disability, compared to those who were started very late in the disease process. $^{29}$

Most patients require symptomatic therapy within 48 months of symptom onset. ${ }^{30}$ The most common reasons given for therapy initiation include development of gait and balance problems, worsening of bradykinesia, and tremor so job security is threatened, or decline in ability to perform activities of daily living. ${ }^{31}$

Starting levodopa in de novo patients results in approximately a $30 \%$ improvement in the UPDRS. ${ }^{11,32,33}$ Benefit is most significant in rigidity and bradykinesia. Patients typically can be managed on $300-600 \mathrm{mg} /$ day in the first two to six years with little need for upward dose adjustment. With disease progression, the level of disability returns to the pretreatment baseline within five years. The benefit of levodopa in PD is usually so significant that lack of responsiveness has implications for diagnosis, i.e. the individual likely has another form of Parkinsonism.

A recurring question is whether initiation of levodopa should be delayed, and if early initiation promotes development of dyskinesias, motor fluctuations, and/or alters disease progression. $^{34,35}$ A number of retrospective studies have suggested that disease progression and development of motor fluctuations is dependent on disease duration, rather than duration or dose of levodopa exposure. ${ }^{36-39}$ One study showed that the effect on prolongation of life expectancy is more pronounced with earlier initiation of levodopa. ${ }^{28}$

To answer this question, a prospective double-blind randomized controlled trial was recently organized by the Parkinson Study Group. The ELLDOPA study ${ }^{5}$ enrolled 360 de novo patients and randomized them to three doses of levodopa/carbidopa (150/37.5mg, 300/75mg and 600/150mg) and placebo. Patients were evaluated over a 40-week period followed by a two-week washout. The study was the first doubleblind controlled trial to demonstrate a dose-related improvement in the motor features of PD in response to levodopa. After the two-week washout, the levodopa treated patients, particularly in the $600 \mathrm{mg}$ group, remained significantly better than those receiving placebo. This suggests that levodopa may have reduced disease progression, although, it is possible that the twoweek wash out was simply not long enough to eliminate a very long pharmacological effect. A smaller subgroup of patients underwent, $\beta$-CIT SPECT scanning (see discussion of Dr. Stoessl). Here, in contrast to the clinical results, levodopa was associated with a greater decline in striatal dopamine transporter function than placebo. Once again, it is not possible to differentiate a negative effect on disease progression, which was certainly not supported by the clinical results, from a direct effect on the uptake of the ligand unrelated to changes in the disease process. Thus, current recommendations are that patients should be started on dopaminergic therapy such as levodopa when disease progression starts to result in disability, in order to experience maximum benefit. ${ }^{40}$ Dyskinesias (typically very mild) developed in approximately $16 \%$ of patients in the highdose group (600 mg/day) after only nine months of treatment. This emphasizes the need to pursue methods of delaying the motor complications of levodopa at the same time as trying to evaluate whether the results of the ELLDOPA trial favor early introduction of levodopa for disease modification.

With disease progression, effectiveness of levodopa diminishes. Initially, an overall loss of benefit is seen, followed by development of wearing off. The patient develops cramping in the legs during the night ("off" dystonia). Each dose of levodopa lasts for a shorter duration of time. ${ }^{41}$

Dyskinesias begin to occur. The prevalence and incidence of motor complications (dyskinesias, fluctuations) has been the subject of many studies. Somewhat different results have been obtained, due to differences in methodology, and definitions of motor complications. Overall, they have been reported to develop in $20-59 \%$ of patients at five years. ${ }^{11,32,33,42}$

Are some formulations of levodopa preferable to others with respect to improving symptomatic control and decreasing rate of complications? Two long-acting (continuous release) preparations are currently available, Sinemet $\mathrm{CR}$, and Madopar HBS®. It has been suggested that use of these preparations will result in continuous dopaminergic receptor stimulation and result in later development of motor complications, as compared to standard preparations.

The first multicentre study ${ }^{43}$ compared Madopar HBS® to standard Madopar in 134 de novo patients over a five-yearperiod in a randomized double-blind parallel-group design. No significant differences were found in daily dose of levodopa, number of doses, or therapeutic benefit at five years between the two preparations. Using the UPDRS Part IV scale, almost $60 \%$ of patients experienced wearing off, up to $20 \%$ experienced early morning dystonia and $41 \%$ had dyskinesia within five years in each group.

The second study ${ }^{32}$ compared Sinemet ${ }^{\circledR}$ vs. Sinemet CR®. This international randomized, double-blind study enrolled 618 patients. At the end of five years, the dose of levodopa and rate of fluctuations and dyskinesia showed no significant difference between the two groups. The rate of motor complications (fluctuations and dyskinesias) was $21-22 \%$. Thus, long acting levodopa preparations did not show an advantage over standard levodopa in postponing or reducing motor fluctuations. The 
lower incidence of motor complications in the second study likely reflects the differences in collecting the information. It should be emphasized that the infrequent dosing used in both studies would not have been expected to eliminate pulsatile stimulation of dopamine receptors which may be critical to the development of motor complications. Thus, study methodology may have largely precluded obtaining a positive result since dosing at a minimum of four to six times per day with a controlled-release preparation would have probably been required to truly provide a more continuous form of drug administration.

Another way of prolonging duration of action of levodopa is by adding a COMT inhibitor such as entacapone. Entacapone acts peripherally, decreasing degradation of levodopa, thus increasing availability centrally. The combination has been shown to be effective in increasing "on" time and decreasing "off" time in PD patients with advanced disease. ${ }^{44}$ The question of whether the initiation of levodopa with COMT inhibition will result in a reduced incidence of motor complications has been addressed by Dr. Olanow in the previous section.

Does addition of entacapone to stable non-fluctuating patients on levodopa result in any benefit? The UK-Irish study ${ }^{45}$ studied both fluctuating and non-fluctuating subjects over a six-monthperiod in a blinded fashion. One hundred and twenty eight nonfluctuating patients were enrolled, with the primary efficacy measure being part II of the UPDRS (activities of daily living). Activities of daily living (ADL) scores showed a small but significant improvement in the entacapone group (10.6 to 10.0 points) compared to a worsening in the placebo group (9.4 to 9.5 points) at a mean interval of four months. The total daily dose of levodopa increased by $40 \mathrm{mg}$ in the placebo group with no significant increase in the entacapone group.

In the CELOMEN study, ${ }^{46}$ PD patients, mostly with advanced disease, were studied for six months to determine safety and efficacy of entacapone as compared to placebo. Only 13\% (41 out of 300) were non-fluctuating. In this small cohort, patients treated with entacapone showed a small improvement in both ADL and motor unified Parkinson's disease rating scale (UPDRS) (1 and 2.3 points respectively), while the placebo group showed a slight deterioration (1.5 and 2.1 points respectively). A decrease of $24 \mathrm{mg}$ of total daily dose of levodopa was seen in the entacapone group. Results did not reach statistical significance most likely due to small sample size.

In the US-01 study ${ }^{47}, 750$ levodopa-treated, stable (nonfluctuating) patients were enrolled in a prospective, doubleblind, multicentre 26-week study. Patients were randomized to receive either entacapone or placebo with each dose of levodopa. Levodopa dose increase was not permitted and the primary outcome measure was change in the UPDRS motor score from baseline. No significant change was seen in the primary outcome measure in the two groups. However, the entacapone group showed a statistically significant improvement in a variety of measures of quality of life including PDQ-39, and SF-36. Also, a larger number of placebo patients required levodopa rescue.

Thus, addition of entacapone to PD patients with early disease may result in improvement in quality of life and ADL, without improvement in motor function. However, caution in interpretation of these results should be used, as there were a large number of dropouts during the trial. The question remains whether use of COMT inhibition with levodopa in de novo patients results in delay in motor complications, and further studies to answer this important question are needed.

\section{Dopamine Agonists in Early Parkinson's Disease}

Janis Miyasaki

Morbidity and mortality in Parkinson's Disease are dramatically reduced with levodopa ${ }^{48}$ (as discussed by Dr. Suchowersky). However, levodopa treatment is also associated with motor complications. Motor complications such as wearing off, on-off phenomena, sudden offs and freezing - and dyskinesias including painful dystonia affect approximately $70 \%$ of patients within five years of starting levodopa. ${ }^{49}$ A study of the impact of dyskinesias over the first four years of dopaminergic therapy did not show a significant negative impact on quality of life by dyskinesias. ${ }^{50}$ The impact of dyskinesias over the longer term is unclear.

Dyskinesias themselves can cause disability through interference with the performance of activities of daily living and indeed they are the major reason for consideration of surgical procedures for PD. ${ }^{51}$ Therefore, attention has turned from symptom control alone to the need to provide satisfactory symptom control and avoid the onset of motor complications. Although the exact molecular basis for these motor complications is controversial, pulsatile stimulation of dopamine receptors by short-duration drugs such as levodopa is felt to be key $^{52,53}$ (as discussed by Dr. Olanow). Hence, even dopamine agonists can induce motor complications if their duration of action is short. Available dopamine agonists such as bromocriptine, pergolide, pramipexole and ropinirole have longer halflives. Of note, the dopamine agonist with the longest half-life, cabergoline, is not available in Canada for the treatment of PD.

Beyond avoidance of motor complications, dopamine agonist therapy theoretically could be associated with a slower progression of illness than levodopa. This theory is partially based on the assumption that levodopa treatment accelerates the progression of $\mathrm{PD}^{54}$. In this paradigm, dopamine is metabolized to toxic-free radical species. Therefore, levodopa treatment further increases dopamine turnover and hence increases free radical formation thereby causing further nigral cell death. ${ }^{55}$ Dopamine agonists permit the use of lower doses of levodopa, thus acting as sparing agents and theoretically reducing the free radical burden. Furthermore, dopamine agonists reduce dopamine formation and turnover through D2 autoreceptor stimulation and further reduce free radical formation. Dopamine agonists may also have direct antioxidant properties and effects on mitochondrial membranes. ${ }^{56}$ Indeed, both pramipexole and ropinirole are proposed as potential neuroprotective drugs for further evaluation in Parkinson's disease by the National Institutes of Health. ${ }^{57}$

The reader is directed to an evidence-based practice parameter dealing with the initiation of treatment in early disease published by the American Academy of Neurology in $2001 .^{40}$ Since this was completed prior to the follow-up publication of the pramipexole study and recent reports describing problematic non-motor complications of Parkinson's disease, here we will concentrate on an update of the pivotal trials evaluating longer term treatment of Parkinson's disease with dopamine agonists 
published in peer-reviewed journals and studies describing side effects of treatment.

\section{Pramipexole}

A four-year study compared pramipexole to levodopa/ carbidopa (CALM-PD) ${ }^{58}$ Patients symptomatic for less than seven years were randomized to pramipexole or levodopa therapy. Assigned drug was escalated over ten weeks. After this time, further symptomatic benefit required the addition of openlabel levodopa. For the final 1.5 years of the study, subjects could increase or decrease the dose of their study drug, or add sustained-release levodopa, amantadine or a COMT inhibitor in addition to open-label levodopa in an effort to replicate accepted practice. The primary outcome variable was the time to development of any motor complication (wearing off, dyskinesias, on-off fluctuations or freezing). The secondary outcome variables were changes in scores for the UPDRS, two quality of life scales (PDQUALIF and EuroQol Visual Analog Scale) and time to require open-label levodopa.

Three hundred and one patients participated in the study. Baseline characteristics were similar in the two groups. A significantly higher number of patients in the pramipexole group required open-label levodopa (72\%) compared with 59\% in the levodopa group. The primary outcomes were statistically different in favour of pramipexole with only $52 \%$ of the pramipexole group reaching endpoint compared with $74 \%$ of the levodopa group for dyskinesias and wearing off.

Secondary outcome variables showed improved UPDRS scores for levodopa compared with pramipexole in all domains (mental status, activities of daily living, motor scores). Quality of life variables did not reveal a difference between pramipexole and levodopa treated subjects.

A parallel study used single photon emission computed tomography (SPECT) brain imaging using the dopamine transporter molecule beta CIT. ${ }^{59}$ Patients were scanned at baseline and at regular intervals (22, 34, 46 months of treatment). Patients randomized to levodopa had greater declines in ligand binding. This may indicate greater loss of striatal dopamine terminals or that initial therapy with dopamine agonists modulates the dopamine transporter rather than conferring neuroprotective benefit (these issues are discussed in greater detail by Dr. Stoessl). Further, UPDRS scores were similar in the levodopa and pramipexole group after 46 months, making neuroprotection unlikely.

\section{Ropinirole}

A five year study of ropinirole versus levodopa for early treatment of Parkinson's disease demonstrated significantly greater motor benefit in subjects randomized to levodopa. ${ }^{10}$ There was no significant difference between the treatment groups at five years based on the activities of daily living portion of the UPDRS. The absolute risk reduction for dyskinesias after five years of treatment was $26 \%$ for the ropinirole group. If disabling dyskinesias were considered alone, the absolute risk reduction was $14 \%$ in the ropinirole group. The number needed to treat with ropinirole monotherapy was seven in order to avoid the development of dyskinesias.

Conclusions: Motor benefit and complications of treatment
Motor benefit therefore is superior for levodopa compared with dopamine agonists in monotherapy use. However, the risk of developing motor complications is far greater with levodopa. In the short term (four years), dyskinesias do not reduce quality of life. Although these studies examined an important clinical question, other questions arise from close examination. Perhaps the difference in rates of motor complications relates directly to the degree of motor benefit obtained. If dopamine agonist motor benefit was equivalent to levodopa, would motor complications still be reduced with dopamine agonist monotherapy? Further, what would different treatment strategies achieve? For instance, if patients received initial dopamine agonist therapy and then used levodopa "rescue" for further motor benefit, would this be superior to levodopa initiation followed by dopamine agonist "rescue"? That is, would either strategy be superior to monotherapy in providing optimal motor benefit and a reduced or acceptable risk of motor complications? Finally, what is the effect of dyskinesias on quality of life in the longer term? Will this new treatment paradigm reduce the number of patients requiring surgery for control of symptoms and dyskinesias?

Another issue to examine is the cost to the patient in terms of non-motor side effects.

\section{Non-motor side effects of anti-parkinsonian treatment}

Common side effects of antiparkinsonian treatment include nausea, vomiting and orthostatic hypotension. Based on the above studies pramipexole caused more somnolence, hallucinations and generalized and peripheral edema compared with levodopa. Ropinirole compared with levodopa induced more hallucinations ( $17 \%$ vs $6 \%$ ), leg edema (14\% vs $6 \%$ ) and somnolence ( $27 \%$ vs $19 \%)$. However, ropinirole and levodopa had similar over all adverse event rates and equivalent drop out rates due to complications.

Subsequent to these studies, the risk of sudden onset of sleep became apparent. The initial report entitled "Falling Asleep at the Wheel" described eight patients treated with pramipexole and one patient, subsequently switched to ropinirole, experiencing sudden falling asleep while driving. ${ }^{60}$ This report used the term "sleep attacks" since the patients reported no prior daytime somnolence and sudden, unavoidable onset of sleep while driving. Subsequent investigators argued that somnolence did not occur without warning but patients ignored cues of somnolence; therefore the term "attack" was inappropriate. ${ }^{61}$ In addition, legal ramifications of automobile accidents gave patients incentive not to recall somnolence prior to the accident. One centre has gone so far as to state that daytime somnolence is an integral feature of Parkinson's disease. ${ }^{62}$ Nonetheless, somnolence is still underrecognized in Parkinson's disease and can increase with antiparkinsonian treatment. Although most patients falling asleep do so with the usual warnings, rare patients have been documented to demonstrate more rapid transitions from wakefulness to deeper stages of sleep. ${ }^{63}$ Levodopa is least likely to cause sudden onset of sleep while cabergoline, pramipexole and ropinirole uncommonly cause sudden onset of sleep.

Although newer dopamine agonists are not ergot based, they seem to cause leg swelling and edema in a significant portion of patients. Recent reports indicate cardiac valvular fibrosis occurs 
with pergolide use ${ }^{64,65}$. In those taking pergolide, $5 \mathrm{mg} /$ day or more, $33 \%$ had significant valvular fibrosis. In lower doses, $19 \%$ of patients had important restrictive valvular heart disease. ${ }^{65}$ In the control group, no patients had valvular heart disease. Therefore, patients requiring higher doses of pergolide should be monitored for occurrence of cardiac valvulopathy. This may be at least partially reversible following pergolide withdrawal. There is no consensus on the best methods of screening for the presence of restrictive valvulopathy in at-risk patients or whether the benefit of maintaining therapy with pergolide or another ergot dopamine agonist justifies the risk in patients whose symptoms are currently well-controlled. A logical approach would be to have an open discussion with the patient explaining the nature of the problem and the current status of uncertainty. Patients should undergo screening echo-cardiography. Patients wishing to be taken off the ergot should be switched to alternative treatment independent of the presence of valvular changes. If abnormalities consistent with restrictive valvulopathy are found on echocardiography, patients should be withdrawn from the ergot agent and switched to a non-ergot agonist or another alternative therapy. This new concern needs to be factored when considering an ergot as the first-line dopamine agonist in the treatment of Parkinson's disease.

\section{Conclusions: Non-motor side effects}

Non-motor side effects of antiparkinsonian treatment are common and significant. The incidence of hallucinations, generalized and peripheral edema are greater for dopamine agonists than levodopa. Although somnolence can occur with all antiparkinsonian treatment or even prior to treatment, levodopa is the least likely to cause more problematic excessive daytime somnolence that occasionally manifests as "sudden onset of sleep" while driving. Cardiac valvulopathy and other fibrotic complications of ergot-derived dopamine agonists are an increasing concern.

\section{Which drug to start with - Dopamine agonists or levodopa?}

An economic study of pramipexole compared with levodopa demonstrated cost savings with pramipexole monotherapy. ${ }^{66}$ The main benefits were avoidance of motor complications and reduced requirement for costly surgery. However, dyskinesias do not have impact in early stages of illness on quality of life. Their impact in later stages of illness remains unclear. Further, the costs of other non-motor side effects were likely underestimated since, with expanded dopamine agonist use, other side effects such as punding and gambling are increasingly reported. ${ }^{67-71}$

At this time, when initiating symptomatic treatment with either a dopamine agonist or levodopa, the physician needs to consider the risk of dyskinesias to the individual patient, their requirement for rapid symptom control and their ability to tolerate dopamine agonists. Therefore, patients at high risk of hallucinations (e.g. cognitive compromise) or daytime somnolence as predicted by the Epworth Sleepiness Scale and possibly obsessive-compulsive behaviour should probably be started on levodopa rather than a dopamine agonist. Although well-designed and executed trials examine discrete scientific questions and evidence-based reviews can synthesize the best available information for specific patient populations and situations, the decision for individual patients takes a myriad of uncontrolled patient characteristics into account and thus remains the privileged domain of the individual clinician and his or her patient.

\section{Functional Imaging Studies in Early Parkinson's Disease}

\section{$J$ Stoessl}

Functional imaging studies can potentially be of enormous use in studying the natural history and progression of Parkinson's disease. Other potential applications include assistance in early diagnosis (particularly if disease modifying therapies become available) and understanding the pathogenesis of longterm complications of therapy. However, the interpretation of these studies can be a minefield, and it is important for anyone reviewing the results to understand the potential pitfalls.

Several approaches are available to assess the integrity of the nigrostriatal dopamine system. The majority of these measure some function that is relatively specific to dopamine neurons. Thus, 6-[ $\left.{ }^{18} \mathrm{~F}\right]$ fluoro-L-dopa (6FD) is taken up by monoaminergic neurons, decarboxylated to $6-\left[{ }^{18} \mathrm{~F}\right]$ fluoro-L-dopamine (6FDA) and 6FDA activity is trapped in synaptic vesicles. If decarboxylation activity is subnormal, or there is reduced capacity to trap 6FDA, the uptake (usually measured as a graphically determined uptake constant, $\mathrm{Ki}$ or Kocc) will be reduced. This has traditionally been regarded as the gold standard for assessing the integrity of the dopamine (DA) system, particularly as 6FD uptake has been shown to correlate with nigral DA cell counts in humans ${ }^{72}$ and in monkeys with MPTP-induced parkinsonism. ${ }^{73}$ The membrane dopamine transporter can be labeled with a number of positron-emitting $\left(\left[{ }^{11} \mathrm{C}\right]\right.$ or $\left[{ }^{18} \mathrm{~F}\right]$-labeled) compounds, or with $\gamma$-emitters for single photon emission computed tomography (SPECT). The majority of these compounds are tropanes (cocaine derivatives), although another option is the positron emitting $\left[{ }^{11} \mathrm{C}\right] d$-threomethylphenidate. ${ }^{74}$ Finally, the vesicular monoamine transporter type 2 (VMAT2) can be labeled using $\left[{ }^{11} \mathrm{C}\right]$ dihydrotetrabenazine (DTBZ) ${ }^{75}$ Imaging studies with any of these tracers show marked (approximately 40\%) reductions in early PD, with a characteristic pattern in which the posterior striatum is affected more than the anterior striatum, in an asymmetric fashion (as is characteristic of the clinical features). Although it was initially hoped that this pattern might help differentiate idiopathic PD from other "Parkinson-plus" syndromes, ${ }^{76}$ it has become apparent over time that this differentiation is not reliable, and the rostro-caudal gradient seen in Parkinson's Disease may also be seen in multiple system atrophy, ${ }^{77}$ and in parkinsonism associated with viral encephalitis ${ }^{78}$ and spinocerebellar atrophy type $2 .{ }^{79}$ It should be noted that in the latter disorder, raclopride binding to dopamine D2 receptors may be increased with a rostrocaudal gradient complementary to the reduction of 6FD uptake, in contrast to multiple system atrophy. 6FD uptake can be used to detect preclinical abnormalities in subjects exposed to MPTP $^{80}$ or in asymptomatic individuals with a genetically determined risk of PD who ultimately go on to develop clinical manifestations. ${ }^{81}$ All of these tracers show abnormalities in the clinically unaffected striatum in patients with clinically unilateral PD. ${ }^{82-85}$

Another approach to functional imaging is the study of changes in regional cerebral glucose metabolism. Although 
traditional comparisons of specific regions may be relatively unrewarding in PD, the application of Principal Components Analysis to the data may reveal altered patterns of connectivity that can be quite specific to PD (or at least levodopa-responsive parkinsonism) and not seen in other disorders that result in parkinsonism. ${ }^{86}$.

Attention has been focused on the merits and pitfalls of functional imaging studies in PD in the last couple of years because of studies in which the imaging outcome measures suggested that certain treatments might modify disease progression, while the clinical data either failed to support this, or indeed suggested changes in the opposite direction. The potential usefulness of a biomarker of disease progression is clear: clinical measures of PD progression are subject to many problems in interpretation. These include confounds related to unanticipated symptomatic effects of the intervention (as demonstrated so well by the DATATOP study ${ }^{87-89}$ ), difficulties achieving full washout of the intervention (as seen in DATATOP and, as well, possibly in the recent ELLDOPA study ${ }^{5}$ ) and the often poor correlation between nigral cell counts and clinical function. Clinical measures are subject to considerable variance, and this may result in the need to study large numbers of subjects in order to detect a statistically significant effect of the intervention, particularly as there may be a large placebo effect in PD. ${ }^{90}$

In the CALM-PD study (as discussed by Dr. Miyasaki), a subgroup of patients who were randomized to receive initial treatment with either levodopa or pramipexole had SPECT scans with the DAT marker $\left[{ }^{123} \mathrm{I}\right] \beta$-CIT. The rate of decline in $\left[{ }^{123} \mathrm{I}\right] \beta$ CIT uptake was greater in patients treated with levodopa than in those treated with pramipexole. However, clinical measures of parkinsonism (assessed 12 hours off medication) favoured levodopa at two years, and were not different between groups at later time points. ${ }^{59}$ Apart from this striking paradox, there were other difficulties with the interpretation of the results. First, the major difference between the groups seemed to occur between baseline and two years, after which time tracer uptake appeared to decline in parallel. It has been suggested that this may reflect a confounding pharmacological effect of the treatment on the uptake of $\left[{ }^{123} \mathrm{I}\right] \beta$-CIT. Furthermore, when the identical two-year data were reported in an earlier publication, there was no difference in the rate of decline of $\left.{ }^{123} \mathrm{I}\right] \beta$-CIT uptake between the two groups ${ }^{11}$. This disparity was thought to perhaps reflect an interval change in the method used to reconstruct the scans.

In the REAL-PET study, 6FD uptake was measured at baseline and after two years of treatment in PD patients who were randomized to receive either levodopa or ropinirole. 6$\left[{ }^{18} \mathrm{~F}\right]$ fluoro-L-dopa (6FD) uptake declined at a significantly faster rate in the levodopa treated group than in those patients who were treated with the dopamine agonist. As was also the case in the CALM-PD study, however, clinical evaluations of parkinsonism (performed with patients on medication) favoured levodopa treatment, although the incidence of dyskinesias was much lower in the ropinirole treated group ${ }^{91}$ (see discussion by Dr. Miyasaki for details). An important methodological distinction between this and the CALM-PD study was the delay of the baseline scan until after active treatment had been initiated, an attempt to minimize the confound arising from potential effects of medication on tracer uptake.
Although both the CALM-PD and REAL-PET studies suggested on the basis of imaging outcomes that the rate of disease progression was slower when treatment was initiated with a dopamine agonist, the clinical measures did not substantiate this and most neurologists remain unconvinced. Detailed analyses of the results and potential confounds are provided elsewhere. ${ }^{92,93}$

Two other recent observations have cast further doubt on the utility of functional imaging measures for the assessment of disease modifying treatments. In the ELLDOPA study (as discussed by Dr. Suchowersky), three doses of levodopa were compared to a placebo. Medications were then withdrawn for two weeks and disease progression over 40 weeks was assessed based on clinical measures. Somewhat unexpectedly, there was a dose-dependent effect in which the rate of clinical decline was apparently lower in patients treated with levodopa. ${ }^{5}$ Although this could be interpreted as evidence of neuroprotection by levodopa, possibly a more likely explanation is inadequate washout, even after two weeks. The issue of concern here is that the imaging data using $\left[{ }^{123} \mathrm{I}\right] \beta-C I T$ SPECT once again were in complete conflict with the clinical observations. The imaging studies suggested a dose dependent increase in the rate of decline of $\left[{ }^{123} \mathrm{I}\right] \beta$-CIT uptake, when analysis was confined to subjects with abnormal uptake at baseline (i.e. subjects with normal scans at baseline were excluded - see below).

Another source of concern comes from recent studies of fetal transplantation for PD. In one of these, the clinical benefits were at best modest, while PET showed robust improvements in 6FD uptake. ${ }^{94}$ In the second study, there was no significant improvement in motor function at two years, while PET showed a sustained and substantial improvement in 6FD uptake, albeit to subnormal levels. ${ }^{95}$

Finally, each of the early treatment studies discussed above (CALM-PD, REAL-PET, ELLDOPA) showed a surprisingly high (close to $15 \%$ ) incidence of people who had been diagnosed with PD but in whom imaging was normal. The meaning of this phenomenon (referred to as SWEDD, or Scans Without Evidence of Dopaminergic Deficit) is as yet unresolved. However, follow-up imaging in these patients shows no evidence of progression, suggesting that they represent an entirely different patient category from typical PD. Some of these patients may have been misdiagnosed and may suffer from essential tremor, an error that is fairly easy to make, particularly in early stages of disease. Others may have a form of parkinsonism unrelated to dopamine deficiency. Tremorpredominant PD may reflect serotonergic more than dopaminergic abnormalities, ${ }^{96}$ although even patients with isolated resting tremor typically demonstrate functional imaging changes compatible with dopamine deficiency.

Taking these sobering observations together, should one conclude that functional imaging should be discarded as a meaningful outcome measure to determine the effects of disease modifying therapies? At this time, such a conclusion would be unduly harsh and defeatist. A number of recent papers have examined the ideal requirements for a biomarker to assess disease progression in PD. ${ }^{97,98}$ Although positron emission tomography (PET) or SPECT may satisfy the majority of the proposed criteria, there is still uncertainty over whether changes are specific to the outcome of interest (in this case loss of 
dopaminergic neurons), or whether other factors (such as compensatory changes or medications) might modify the measurement. It appears clear that 6FD, DAT ligands and DTBZ do not decline to exactly the same degree in $\mathrm{PD}^{85}$. This may reflect compensatory downregulation of the DAT and upregulation of L-aromatic amino acid decarboxylase, in an effort to maintain dopamine levels in the synapse. The effects of medication on the expression of these markers are unclear. While some authors have reported changes in DAT expression following a short course of dopaminergic therapy in PD, ${ }^{99,100}$ this is inconsistent, and it has been difficult to demonstrate changes in 6FD uptake in response to medications. ${ }^{101}$ DTBZ may be advantageous in this respect, as the VMAT2 appears to be relatively resistant to the effects of medication. ${ }^{102-104}$

Clinicians will understandably be hesitant to accept an effect of a therapeutic intervention that can be demonstrated on a test only, if there is no clinical correlate. However, it should be remembered that the clinical outcomes employed may also be suboptimal. Thus, the UPDRS Motor scale may be unduly influenced by measures of tremor or rigidity, whereas a measure that focuses entirely on bradykinesia may provide the best marker of dopaminergic deficiency. ${ }^{105}$

\section{List of Participants}

Robert Chen, (Toronto), Martin Cloutier, (Montreal), Wendy Galpern, (Fellow, Toronto), Mark Guttman, (Markham), David Grimes, (Ottawa), Serena Hung, (Fellow, Toronto), Anne-Louise Lafontaine (Montreal), Anthony E. Lang (Organizer and Chair, Toronto), Connie Maras, (Toronto), Calvin Melmed, (Montreal), Janice Miyasaki, (Speaker, Toronto), C. Warren Olanow (Speaker, New York), Michel Panisset, (Montreal), Jean Rivest, (Sherbrooke), A. Jon Stoessl, (Speaker, Vancouver), Oksana Suchowersky, (Calgary), Ayse Tokcaer, (Observer, Toronto), Joseph Tsui, (Vancouver),

\section{ACKNOWLEDGMENT}

This forum was sponsored by an unrestricted educational grant from Novartis, Canada. None of the authors have any conflicts of interest related to the material presented in this document.

\section{REFERENCE LIST}

1. Lang AE, Lozano AM. Parkinson's disease - First of two parts. N Engl J Med 1998; 339:1044-1053.

2. Olanow CW, Obeso JA. Preventing levodopa-induced dyskinesias. Ann Neurol 2000; 47:S167-S178.

3. Grace AA, Bunney BS. The control of firing pattern in nigral dopamine neurons: single spike firing. J Neurosci 1984; 4:28662876.

4. Schultz W. Behavior-related activity of primate dopamine neurons. Rev Neurol (Paris) 1994; 150:634-639.

5. Fahn S, Oakes D, Shoulson I, et al. Levodopa and the progression of Parkinson's disease. N Engl J Med 2004; 351:2498-2508.

6. Obeso JA, Rodriguez-Oroz MC, Rodriguez M, DeLong MR, Olanow CW. Pathophysiology of levodopa-induced dyskinesias in Parkinson's disease: problems with the current model. Ann Neurol 2000; 47:S22-S34.

7. Bedard PJ, DiPaolo T, Falardeau P, Coucher R. Chronic treatment with L-Dopa, but not bromocriptine induces dyskinesia in MPTPParkinsonian monkeys. Correlation with $[3 \mathrm{H}]$ spiperone binding. Brain Res 1986; 379:294-299.
8. Pearce RK, Banerji T, Jenner P, Marsden CD. De novo administration of ropinirole and bromocriptine induces less dyskinesia than L-dopa in the MPTP-treated marmoset. Mov Disord 1998; 13:234-241.

9. Blanchet PJ, Calon F, Martel JC, et al. Continuous administration decreases and pulsatile administration increases behavioral sensitivity to a novel dopamine D2 agonist (U-91356A) in MPTP-exposed monkeys. J Pharmacol Exp Ther 1995; 272:854859.

10. Rascol O, Brooks DJ, Korczyn AD, et al. A five-year study of the incidence of dyskinesia in patients with early Parkinson's disease who were treated with ropinirole or levodopa. N Engl J Med 2000; 342:1484-1491.

11. Holloway R, Shoulson I, Kieburtz K, et al. Pramipexole vs levodopa as initial treatment for Parkinson disease - A randomized controlled trial. JAMA 2000; 284:1931-1938.

12. Sage JI, Trooskin S, Sonsalla PK, Heikkila R, Duvoisin RC. Longterm duodenal infusion of levodopa for motor fluctuations in parkinsonism. Ann Neurol 1988; 24:87-89.

13. Ruggieri S, Stocchi F, Carta A, Catarci M, Agnoli A. Jejunal delivery of levodopa methyl ester. Lancet 1989; 2:45-46.

14. Salarian A, Russmann H, Vingerhoets FJG, et al. Gait assessment in Parkinson's disease: toward an ambulatory system for long-term monitoring. IEEE Trans Biomed Eng 2004; 51(8):1434-1443.

15. Kurlan R, Rubin AJ, Miller C, et al. Duodenal delivery of levodopa for on-off fluctuations in parkinsonism: preliminary observations. Ann Neurol 1986; 20:262-265.

16. Kurth MC, Tetrud JW, Tanner CM, et al. Double-blind, placebocontrolled, crossover study of duodenal infusion of levodopa/carbidopa in Parkinson's disease patients with 'on-off' fluctuations. Neurology 1993; 43:1698-1703.

17. Nutt JG, Carter JH, Lea ES, Woodward WR. Motor fluctuations during continuous levodopa infusions in patients with Parkinson's disease. Mov Disord 1997; 12:285-292.

18. Nilsson D, Hansson LE, Johansson K, et al. Long-term intraduodenal infusion of a water based levodopa-carbidopa dispersion in very advanced Parkinson's disease. Acta Neurol Scand 1998; 97:175-183.

19. Syed N, Murphy J, Zimmerman T, Jr., Mark MH, Sage JI. Ten years' experience with enteral levodopa infusions for motor fluctuations in Parkinson's disease. Mov Disord 1998; 13:336-338.

20. Stocchi F, Vacca L, Ruggieri S, Olanow CW. Infusion of levodopa methyl ester in patients with advanced PD: a clinical and pharmacokinetic study. Arch Neurol 2004; in press.

21. Olanow CW, Stocchi F. COMT inhibitors in Parkinson's disease Can they prevent and/or reverse levodopa-induced motor complications? Neurology 2004; 62:S72-S81.

22. Nutt JG, Woodward WR, Beckner RM, et al. Effect of peripheral catechol-O-methyltransferase inhibition on the pharmacokinetics and pharmacodynamics of levodopa in parkinsonian patients. Neurology 1994; 44:913-919.

23. Smith LA, Jackson MJ, Al-Barghouthy G, et al. Multiple small dosses of levodopa plus entacapone produces continuous dopaminergic stimulation and reduces dyskinesia induction in MPTP-treated drug naive primates. Mov Disord 2004; in press.

24. Barbeau A, Roy M. Six-year results of treatment with levodopa plus benzerazide in Parkinson's disease. Neurology 1976; 26:399-404.

25. Koller WC, Hubble JP. Levodopa therapy in Parkinson's disease. Neurology 1990; 40(10 Suppl 3):S7.

26. Hoehn MM, Yahr DM. Parkinsonism: Onset, progression and mortality. Neurology 1967; 17:427-442.

27. Bennett DA, Beckett LA, Murray AM, et al. Prevalence of Parkinsonian signs and associated mortality in a community population of older people. N Engl J Med 1996; 334(2):71-76.

28. Yahr M. Evaluation of long-term therapy in Parkinson's disease: mortality and therapeutic efficacy. In: Birkmayer W, Hornykiewicz O, (Eds). Advances In Parkinsonism: Biochemistry, Physiology, Treatment. Basle: Editiones $<$ Roche $>$, 1976: 435-444.

29. Rajput AH, Uitti RJ, Offord KP. Timely levodopa (LD) administration prolongs survival in Parkinson's disease. Parkinsonism \& Rel Disord 1997; 3:159-165. 
30. Goetz CG, Tanner CM, Shannon KM. Progression of Parkinson's disease without levodopa. Neurology 1987; 37:695-698.

31. Parkinson Study Group. DATATOP: A Multicenter Controlled Clinical Trial in Early Parkinson's Disease. Arch Neurol 1989; 46:1052-1060.

32. Koller WC, Hutton JT, Tolosa E, Capilldeo R. Carbidopa LSG Immediate-release and controlled-release carbidopa/levodopa in PD - A 5-year randomized multicenter study. Neurology 1999; 53:1012-1019.

33. Rinne UK, Bracco F, Chouza C, et al. Early treatment of Parkinson's disease with cabergoline delays the onset of motor complications - results of a double-blind levodopa controlled trial. Drugs 1998; 55:23-30.

34. Rajput AH, Stern W, Laverty WH. Chronic low-dose levodopa therapy in Parkinson's disease: an argument for delaying levodopa therapy. Neurology 1984; 34:991-996.

35. Lesser RP, Fahn S, Snider SR, et al. Analysis of the clinical problems in parkinsonism and the complications of long-term levodopa therapy. Neurology 1979; 29:1253-1260.

36. Markham CH, Diamond SG. Evidence to support early levodopa therapy in Parkinson disease. Neurology 1981; 31:125-131.

37. Cedarbaum JM, Gandy SE, McDowell FH. "Early" initiation of levodopa treatment does not promote the development of motor response fluctuations, dyskinesias, or dementia in Parkinson's disease. Neurology 1991; 41:622-629.

38. Caraceni T, Scigliano G, Musicco M. The occurrence of motor fluctuations in parkinsonian patients treated long term with levodopa: role of early treatment and disease progression. Neurology 1991; 41:380-384.

39. Horstink MW, Zijlmans JC, Pasman JW, Berger HJ, Van't Hof MA. Severity of Parkinson's disease is a risk factor for peak-dose dyskinesia. J Neurol Neurosurg Psychiatry 1990; 53:224-226.

40. Miyasaki JM, Martin W, Suchowersky O, Weiner WJ, Lang AE. Practice parameter: initiation of treatment for Parkinson's disease: An evidence-based review - report of the quality standards subcommittee of the American Academy of Neurology. Neurology 2002; 58:11-17.

41. Poewe WH. Clinical aspects of motor fluctuations in Parkinson's disease. Neurology 1994; 44 (Suppl. 6):S6-S9.

42. Parkinson Study Group. Impact of Deprenyl and Tocopherol treatment on Parkinson's Disease in DATATOP subjects not requiring Levodopa. Ann Neurol 1996; 39:29-36.

43. Dupont E, Andersen A, Boas J, et al. Sustained-release Madopar HBS compared with standard Madopar in the long-term treatment of de novo parkinsonian patients. Acta Neurol Scand 1996; 93:14-20.

44. Ruottinen HM, Rinne UK. Entacapone prolongs levodopa response in a one month double blind study in parkinsonian patients with levodopa related fluctuations. J Neurol Neurosurg Psychiatry 1996; 60:36-40.

45. Brooks DJ, Sagar H, UK-Irish Entacapone Study Group. Entacapone is beneficial in both fluctuating and non-fluctuating patients with Parkinson's disease: a randomized, placebo controlled, double blind, six month study. J Neurol Neurosurg Psychiatry 2003; 74:1071-1079.

46. Poewe WH, Deuschl G, Gordin A, Kultalahti ER, Leinonen M. Efficacy and safety of entacapone in Parkinson's disease patients with suboptimal levodopa response: a 6-month randomized placebo-controlled double-blind study in Germany and Austria (Celomen study). Acta Neurol Scand 2002; 105:245-255.

47. Olanow CW, Kieburtz K, Stern M, et al. Double-blind, placebocontrolled study of entacapone in levodopa-treated patients with stable Parkinson disease. Arch Neurol 2004; 61:1563-1568.

48. Uitti RJ, Ahlskog JE, Maraganore DM, et al. Levodopa therapy and survival in idiopathic Parkinson's disease: Olmsted County project. Neurology 1993; 43:1918-1926.

49. Ahlskog JE, Muenter MD. Frequency of levodopa-related dyskinesias and motor fluctuations as estimated from the cumulative literature. Mov Disord 2001; 16:448-458.

50. Marras C, Lang A, Krahn M, et al. Quality of life in early Parkinson's disease: Impact of dyskinesias and motor fluctuations. Mov Disord 2004; 19:22-28.
51. Lozano AM. Surgery for Parkinson's disease, the five W's: why, who, what, where, and when. Adv Neurol 2003; 91:303-307.

52. Juncos JL, Engber TM, Susel Z, et al. Continuous and intermittent levodopa administration differentially affect basal ganglia function. Ann Neurol 1989; 25:473-478.

53. Chase TN, Oh JD. Striatal mechanisms and pathogenesis of parkinsonian signs and motor complications. Ann Neurol 2000; 47:S122-S130.

54. Papa SM, Engber TM, Kask AM, Chase TN. Motor fluctuations in levodopa treated parkinsonian rats: relation to lesion extent and treatment duration. Brain Res 1994; 662:69-74.

55. Mytilineou C, Walker RH, JnoBaptiste R, Olanow CW. Levodopa is toxic to dopamine neurons in an in vitro but not an in vivo model of oxidative stress. J Pharmacol Exp Ther 2003; 304:792-800.

56. Nishibayashi S, Asanuma M, Kohno M, Gomez-Vargas M, Ogawa $\mathrm{N}$. Scavenging effects of dopamine agonists on nitric oxide radicals. J Neurochem 1996; 67:2208-2211.

57. Diguet E, Gross CE, Bezard E, et al. Neuroprotective agents for clinical trials in Parkinson's disease: A systematic assessment. Neurology 2004; 62:158.

58. Holloway RG, Shoulson I, Kieburtz K, et al. Pramipexole vs levodopa as initial treatment for Parkinson disease - a 4-year randomized controlled trial. Arch Neurol 2004; 61:1044-1053.

59. Marek K, Seibyl J, Shoulson I, et al. Dopamine transporter brain imaging to assess the effects of pramipexole vs levodopa on Parkinson disease progression. JAMA 2002; 287:1653-1661.

60. Frucht S, Rogers JD, Greene PE, Gordon MF, Fahn S. Falling asleep at the wheel: motor vehicle mishaps in persons taking pramipexole and ropinirole. Neurology 1999; 52:1908-1910.

61. Olanow CW, Schapira AHV, Roth T. Waking up to sleep episodes in Parkinson's disease. Mov Disord 2000; 15:212-215.

62. Arnulf I, Konofal E, Merino-Andreu M, et al. Parkinson's disease and sleepiness - an integral part of PD. Neurology 2002; 58:10191024.

63. Tracik F, Ebersbach G. Sudden daytime sleep onset in Parkinson's disease: polysomnographic recordings. Mov Disord 2001; 16:500-506.

64. Van Camp G, Flamez A, Cosyns B, et al. Treatment of Parkinson's disease with pergolide and relation to restrictive valvular heart disease. Lancet 2004; 363:1179-1183.

65. Horvath J, Fross RD, Kleiner-Fisman G, et al. Severe multivalvular heart disease: a new complication of the ergot derivative dopamine agonists. Mov Disord 2004; 19:656-662.

66. Noyes K, Dick A, Holloway RG, and the Parkinson Study Group. Pramipexole vs levodopa as initial treatment for Parkinson's disease. A randomized clinical-economic trail. Med Decis Making 2004; 24: 472-485.

67. Fernandez HH, Friedman JH. Punding on 1-dopa. Mov Disord 1999; 14:836-838.

68. Evans AH, Katzenschlager R, Paviour D, et al. Punding in Parkinson's disease: its relation to the dopamine dysregulation syndrome. Mov Disord 2004; 19:397-405.

69. Kurlan R. Disabling repetitive behaviors in Parkinson's disease. Mov Disord 2004; 19:433-437.

70. Voon V. Repetition, repetition, and repetition: compulsive and punding behaviors in Parkinson's disease. Mov Disord 2004; 19:367-370

71. Gschwandtner U, Aston J, Renaud S, Fuhr P. Pathologic gambling in patients with Parkinson's disease. Clin Neuropharmacol 2001; 24:170-172.

72. Snow BJ, Tooyama I, McGeer EG, et al. Human positron emission tomographic [18F]fluorodopa studies correlate with dopamine cell counts and levels. Ann Neurol 1993; 34:324-330.

73. Pate BD, Kawamata T, Yamada $\mathrm{T}$, et al. Correlation of striatal fluorodopa uptake in the MPTP monkey with dopaminergic indices. Ann Neurol 1993; 34:331-338.

74. Volkow ND, Ding YS, Fowler JS, et al. A new PET ligand for the dopamine transporter: studies in the human brain. J Nucl Med 1995; 36:2162-2168.

75. Kilbourn MR. In vivo radiotracers for vesicular neurotransmitter transporters. Nucl Med Biol 1997; 24:615-619. 
76. Brooks DJ, Ibanez V, Sawle GV, et al. Differing Patterns of Striatal ${ }^{18}$ F-Dopa Uptake in Parkinson's Disease, Multiple System Atrophy, and Progressive Supranuclear Palsy. Ann Neurol 1990; 28:547-555.

77. Antonini A, Leenders KL, Vontobel P, et al. Complementary PET studies of striatal neuronal function in the differential diagnosis between multiple system atrophy and Parkinson's disease. Brain 1997; 120:2187-2195.

78. Lin SK, Vingerhoets FJG, Snow B, Schulzer M, Wai YY. Isolated involvement of substantia nigra in acute transient parkinsonism: MRI and PET observations. Parkinsonism \& Rel Disord 1995; $1: 67-73$.

79. Furtado S, Farrer M, Tsuboi $\mathrm{Y}$, et al. SCA-2 presenting as parkinsonism in an Alberta family - clinical, genetic, and PET findings. Neurology 2002; 59:1625-1627.

80. Calne DB, Langston JW, Martin WRW, et al. Positron emission tomography after MPTP: observations relating to the cause of Parkinson's disease. Nature 1985; 317:246-248.

81. Piccini P, Burn DJ, Ceravolo R, Maraganore D, Brooks DJ. The role of inheritance in sporadic Parkinson's disease: evidence from a longitudinal study of dopaminergic function in twins. Ann Neurol 1999; 45:577-582.

82. Frost JJ, Rosier AJ, Reich SG, et al. Positron emission tomographic imaging of the dopamine transporter with 11C-WIN 35,428 reveals marked declines in mild Parkinson's disease. Ann Neurol 1993; 34:423-431.

83. Marek KL, Seibyl JP, Zoghbi SS, et al. [ $\left.{ }^{123} \mathrm{I}\right]$ b-CIT/SPECT imaging demonstrates bilateral loss of dopamine transporters in hemiParkinson's disease. Neurology 1996; 46:231-237.

84. Guttman M, Burkholder J, Kish SJ, et al. [ $\left.{ }^{11} \mathrm{C}\right]$ RTI-32 PET studies of the dopamine transporter in early dopa-naive Parkinson's disease: Implications for the symptomatic threshold. Neurology 1997; 48:1578-1583.

85. Lee CS, Samii A, Sossi V, et al. In vivo positron emission tomographic evidence for compensatory changes in presynaptic dopaminergic nerve terminals in Parkinson's disease. Ann Neurol 2000; 47:493-503.

86. Eidelberg D, Moeller JR, Ishikawa T, et al. Early differential diagnosis of Parkinson's disease with ${ }^{18} \mathrm{~F}$ - fluorodeoxyglucose and positron emission tomography. Neurology 1995; 45:19952004.

87. Parkinson Study Group. Effect of deprenyl on the progression of disability in early Parkinson's disease. N Engl J Med 1989; 321:1364-1371.

88. The Parkinson Study Group. Effects of Tocopherol and Deprenyl on the Progression of Disability in Early Parkinson's Disease. N Engl J Med 1993; 328:176-183.

89. Schulzer M, Mak E, Calne DB. The antiparkinson efficacy of deprenyl derives from transient improvement that is likely to be symptomatic. Ann Neurol 1992; 32:795-798.
90. De la Fuente-Fernàndez R, Stoessl AJ. The placebo effect in Parkinson's disease. Trends Neuro 2002; 25:302-306.

91. Whone AL, Watts RL, Stoessl AJ, et al. Slower progression of Parkinson's disease with ropinirole versus levodopa: The REALPET study. Ann Neurol 2003; 54:93-101.

92. Ahlskog JE. Slowing Parkinson's disease progression - recent dopamine agonist trials. Neurology 2003; 60:381-389.

93. Albin RL, Frey KA. Initial agonist treatment of Parkinson disease a critique. Neurology 2003; 60:390-394.

94. Freed CR, Greene PE, Breeze RE, et al. Transplantation of embryonic dopamine neurons for severe Parkinson's disease. N Engl J Med 2001; 344:710-719.

95. Olanow CW, Goetz CG, Kordower JH, et al. A double-blind controlled trial of bilateral fetal nigral transplantation in Parkinson's disease. Ann Neurol 2003; 54:403-414.

96. Doder M, Rabiner EA, Turjanski N, Lees AJ, Brooks DJ. Tremor in Parkinson's disease and serotonergic dysfunction - An 11C-WAY 100635 PET study. Neurology 2003; 60:601-605.

97. Brooks DJ, Frey KA, Marek KL, et al. Assessment of neuroimaging techniques as biomarkers of the progression of Parkinson's disease. Exp Neurol 2003; 184:S68-S79.

98. DeKosky ST, Marek K. Looking backward to move forward: early detection of neurodegenerative disorders. Science 2003; 302:830-834.

99. Guttman M, Stewart D, Hussey D, et al. Influence of L-dopa and pramipexole on striatal dopamine transporter in early PD. Neurology 2001; 56:1559-1564.

100. Ahlskog JE, Uitti RJ, O'Connor MK, et al. The effect of dopamine agonist therapy on dopamine transporter imaging in Parkinson's disease. Mov Disord 1999; 14:940-946.

101. Ceravolo R, Piccini P, Bailey DL, et al. ${ }^{18} \mathrm{~F}$-dopa PET evidence that tolcapone acts as a central COMT inhibitor in Parkinson's disease. Synapse 2002; 43:201-207.

102. Vander BT, Kilbourn M, Desmond T, Kuhl D, Frey K. The vesicular monoamine transporter is not regulated by dopaminergic drug treatments. Eur J Pharmacol 1995; 294:577-583.

103.Wilson JM, Kish SJ. The vesicular monoamine transporter, in contrast to the dopamine transporter, is not altered by chronic cocaine self-administration in the rat. J Neurosci 1996; 16:35073510 .

104. Kemmerer ES, Desmond TJ, Albin RL, Kilbourn MR, Frey KA. Treatment effects on nigrostriatal projection integrity in partial 6OHDA lesions: comparison of L-DOPA and pramipexole. Exp Neurol 2003; 183:81-86.

105. Vingerhoets FJG, Schulzer M, Caine DB, Snow BJ. Which clinical sign of Parkinson's disease best reflects the nigrostriatal lesion? Ann Neurol 1997; 41:58-64. 